\title{
Comparason of Peri-Articular Multimodal Drug Injection with Femoral and Sciatic Nerve Block after Total Knee Arthroplasty
}

\author{
Calum H. C. Arthur, Alexander M. Wood, Charles Leeson-Payne, Steffen J. Breusch
}

Orthopaedic Department, Royal Infirmary of Edinburgh, Edinburgh, UK.

Email: calumarthur@doctors.org.uk

Received October $9^{\text {th }}, 2012$; revised November $9^{\text {th }}, 2012$; accepted November $23^{\text {rd }}, 2012$

\begin{abstract}
Good perioperative analgesia following total knee arthroplasty facilitates rehabilitation and may reduce hospital stay. We present the patient analgesic requirements and rehabilitation of a prospective single surgeon series after the introduction of a multimodal drug injection during his total knee arthroplasty. Basic epidemiological data as well as analgesic, antiemetic requirements and time to straight leg raised was collected on 27 consecutive patients (group 1) whom received the multimodal drug injection, consisting of levobupivacaine, ketorolac and adrenaline at the time of their total knee arthroplasty under spinal anaesthesia. Their rehabilitation was compared to a retrospective review of patients who were case matched by age and sex (group $2(n=26)$ ), whom had received the unit standard of spinal anaesthetic and a femoral and sciatic block at the time of their operation. Patients in group 1 had significantly lower analgesic and antiemetic requirements than group 2 . Group 1 also had a significantly shorter hospital stay. We have demonstrated that periarticular multimodal drug injection can improve perioperative analgesia and mobilisation following total knee arthroplasty as well as reducing opioid requirements and side effects.
\end{abstract}

Keywords: Analgesia; Total Knee Replacement; Multimodal Drug Injection; Peripheral Nerve Blocks; Rehabilitation

\section{Introduction}

Knee replacement surgery is a major painful operative procedure and adequate perioperative analgesia is crucial for a successful outcome [1,2]. Good perioperative analgesia following total knee arthroplasty facilitates rehabilitation by enabling early ambulation and initiation of physiotherapy [3-5]. Swifter rehabilitation and improved pain relief can lead to a reduction in hospital stay [4-6].

There are numerous methods described for providing analgesia following a major knee operation the most popular include; systemic opioids, neuro-axial techniques (spinal, epidural anaesthesia) and peripheral nerve blocks. Each analgesic method has its advantages and disadvantages. Opiods are excellent analgesics, but they have significant side effects including respiratory depression, nausea, vomiting, pruritus and sedation all of which can prolong rehabilitation. Peripheral nerve block may delay patient mobilisation and therefore rehabilitation.

The concept of a multimodal drug injection is a relatively recent arrival in orthopaedics [7-11]. By administering pre-emptive analgesia directly to the operative site it has been shown to reduce the need for opioid analgesics [6-9] and therefore reduce their side effects [7]. It can negate the need for peripheral nerve block and thereby hasten rehabilitation and improve patient satisfaction $[7,9]$.

A multimodal drug injection which includes local anaesthetic, non-steroidal analgesic and adrenaline was introduced into the operating practice of one arthroplasty surgeon in place of peripheral nerve blockade when performing total knee arthroplasty.

The aim of this study was to compare the rehabilitation and analgesic requirements of patients receiving the multimodal drug injection to patients who had received peripheral nerve blockade.

\section{Patients and Methods}

We prospectively collected data on 27 consecutive patients (group 1) who underwent total knee arthroplasty under the care of the senior author between July 2007 and February 2008.

Data was collected from the patients' notes, drug cardex and Patient Controlled Analgesia (PCA) charts. These patients received a spinal anaesthetic and a multimodal drug injection as listed in Table 1, which was administered at the time of their operation and infiltrated 
Table 1. Contents of Multimodal drug Injection.

\begin{tabular}{cc}
\hline $60 \mathrm{~mL}$ & Levobupivocaine 2.5\% \\
$30 \mathrm{mg}$ & ketorolac \\
$0.5 \mathrm{mg}$ & Adrenaline (1 in 1000) \\
$40 \mathrm{~mL}$ & Normal Saline \\
\hline
\end{tabular}

into the posterior joint capsule and into the joint during the procedure with the remainder infiltrated around the wound after skin closure.

Group $2(n=26)$ were a retrospective group who were operated on by the same surgeon before the introduction of the multimodal drug injection. All had a total knee arthroplasty under spinal anaesthetic but with a femoral and sciatic nerve block. Data for these patients was collected retrospectively from their medical notes.

In all patients the operation was performed by the senior author or under his direct supervision. All implants were the cemented cruciate retaining PFC Sigma total knee replacement. All were performed through a medial parapatellar approach, with tournequet and no postoperative drains were used. Apart from their analgesic regime, all patients underwent an identical care pathway postoperatively.

Basic Epidemiological data was recorded including age, sex, diagnosis and body mass index (BMI).

Post-operative data included PCA requirements, other analgesic requirements, antiemetics administered, time to straight leg raise and length of in patient stay. Due to the amount of different types of opioid analgesics administered to patients all doses were combined to a total morphine equivalent dose using the formula in Figure 1.

Data was analysed using chi-squared tests with Yates' correction for binary variables, chi-squared tests for trend for ordinal variables and two sample t-tests for normally distributed quantitative variables.

Multiple regressions were used to compare the groups after adjusting for age. A $P$-value of 0.05 was used to indicate significance.

\section{Results}

Group 1 contained 10 men and 17 women with a mean age of 70.6 years, and a mean BMI of $29.7 \mathrm{~kg} / \mathrm{m}^{2}$. 24 out of the group had a pre-operative diagnosis of Osteoarthritis, 2 Rheumatoid arthritis and 1 Seronegative Arthroplathy. Group 2 contained 10 men and 16 women with a mean age of 66.1 years and a mean BMI of 32.3 $\mathrm{kg} / \mathrm{m}^{2} .23$ out of the group had a pre-operative diagnosis of Osteoarthritis, 2 rheumatoid arthritis and 1 mixed picture. There was no statistical difference in male:female ratio $(P=1.00)$, BMI $(P=0.09)$ diagnosis $(P=1.00)$ between the two groups. There was a borderline signifi- cantly older patient in group $1(P=0.05)$ (Table 2$)$.

Group 1 had a statistically significantly shorter length of hospital stay 4.6 days compared to 5.3 days $(P=$ 0.016). Group 1 received a significantly lower total morphine equivalents; $207 \mathrm{mg}$ compared to $295 \mathrm{mg}(P=$ 0.005). Group 1 also received a significantly lower number of antiemetics doses; 1.3 compared to $2.8(P=0.04)$. There was no significant difference found between amounts of PCA administered or time to straight leg raise (Table 3).

Because of the borderline significant difference in mean age of the two groups, they were also compared after adjusting for age, and these differences remained significant: indeed the adjusted $P$-value for length of stay dropped to 0.007 , reflecting the fact that the injection groups had shorter stays in spite of being older on average.

There was no intra-operative or in-hospital complications in either group.

\section{Discussion}

There is currently no evidence-based consensus on the optimal postoperative pain management strategy follow-

\begin{tabular}{|l|} 
Dose of Tramadol $\times 0.15$ \\
Dose of Dihydrocodeine $\times 0.3$ \\
Dose of Morphine $\times 1$ \\
Dose of Oxycodone $\times 2$
\end{tabular}

Figure 1. Formula for calculating Total Morphine Equivalents.

Table 2. Pre-operative factors in the two study groups.

\begin{tabular}{cccc}
\hline Factor & Injection & F + S & P-value \\
\hline Age & $70.6(8.6)$ & $66.1(7.8)$ & 0.05 \\
Male sex & $10(37)$ & $10(38)$ & 1.00 \\
BMI & $29.7(4.6)$ & $32.3(6.3)$ & 0.09 \\
Diagnosis OA & $24(88)$ & $23(88)$ & 1.00 \\
\hline
\end{tabular}

a. Figures shown are mean (SD) or number (\%).

Table 3. Outcomes in the two study groups.

\begin{tabular}{cccc}
\hline Outcome & Injection & F + S & P-value \\
\hline Length of stay (days) & $4.6(0.9)$ & $5.3(1.4)$ & 0.016 \\
Total PCA (mg) & $29.6(31.5)$ & $34.7(29.5)$ & 0.15 \\
Total morphine equivalent & $207(118)$ & $295(100)$ & 0.005 \\
Total doses antiemetic & $1.3(1.1)$ & $2.8(2.3)$ & 0.004 \\
Time to SLR (days) & $2.6(1.6)$ & $3.3(1.8)$ & 0.15 \\
\hline
\end{tabular}

a. Figures shown are mean (SD) or number (\%). 
ing total knee arthroplasty [10]. Early postoperative recovery and mobilisation is improved by effective pain control. A recent systematic review looked at the evidence for different strategies and had limited recommendations mainly due to the lack of quality studies [10]. Its main guidance included the use of femoral nerve blocks or spinal local anaesthetics with an opioid. It also recommended the postoperative use of strong opioids in combination with non-opioid analgesics [10].

Because of this lack of quality evidence there is considerable variation in postoperative analgesic regimes, which are influence by many different factors such as local experience and skills as well as surgeon preference. Epidural analgesia is regarded by some as the gold standard for pain relief for total knee arthroplasty [12], however there is also considerable support for femoral and sciatic nerve blocks. Peripheral nerve blockade is has a good evidence base [10,12], however this treatment includes a motor blockade, which may delay mobilisation and as a consequence of persistent quadriceps weakness it has been shown to have a increased risk of serious postoperative falls [13].

It has been well documented that local anaesthetic injection following knee surgery can reduce the need for opioid analgesics [14,15] and one study found that the administration of local anaesthetic injection following knee arthroscopy led to a shorter hospital stay [15].

We used Ketorolac in our study as it has been demonstrated that when Ketorolac is added to local anaesthetic there is an improvement in its analgesic efficacy following knee arthroscopy [16]. It has also been used in total hip arthroplasty in combination with ropivacaine and adrenaline and was shown to be effective at reducing the immediate pain relief for up to 2 weeks postoperatively with better function at 1 week [17].

Adrenaline was used in our injection to improve the duration of local blockade and to reduce post-operative bleeding. The use of adrenaline in total knee arthro plasty has previously been shown to decrease the ammount of bleeding and transfusion requirement when infiltrated prior to surgical incision [18]. Although in our study we did not look at post-operative bleeding or transfusion requirements.

There has been recent additional support for the use of local infiltration of analgesia following total knee replacement where a review of the current literature [19] concluded that this can provide improved post-operative pain relief, although it did conclude that there was no clear evidence that it reduced hospital stay.

Our study has shown that not only has our multimodal drug injection reduced the need for narcotic analgesics and therefore reduced their side effects it has also led to a reduction in hospital stay and a trend towards swifter re- habilitation.

Whilst our study is retrospective and does not include patient pain or satisfaction scores, it does demonstrate a significant reduction in post operative opioid requirement, antiemetic usage and a resultant decrease in inpatient stay. There is a requirement to run a prospective randomized study to confirm the benefits of a multimodal interoperative drug injection whilst performing total knee arthroplasty.

\section{Conclusion}

Periarticular multimodal drug injection can reduce the requirement for opioid analgesics and hence reduce their side effects. It also helps reduce hospital stay when compared to a standard spinal anaesthesia with femoral and sciatic nerve blocks. We believe this should improve the cost-effectiveness of total knee arthroplasty and reduce the sequelae of long in-patient stays post operation.

\section{REFERENCES}

[1] A. S. Malik, O. Boyko, N. Atkar and W. F. Young, “A Comparative Study of MR Imaging Profile of Titanium Pedicle Screws,” Acta Radiologica, Vol. 42, No. 3, 2001, pp. 291-293. doi:10.1080/028418501127346846

[2] A. Buvanendran, J. S. Kroin, K. J. Tuman, T. R. Lubenow, D. Elmofty, M. Moric and A. G. Rosenburg, "Effects of Perioperative Administration of a Selective Cycloxygenase 2 Inhinitor on Pain Management and Recovery of Function after Knee Replacement," Journal of the American Medical Association, Vol. 290, No. 18, 2003, pp. 2411-2418. doi:10.1001/jama.290.18.2411

[3] F. J. Singelyn, M. Deyaert, D. Joris, E. Pendeville and J. M. Gouverneur, "Effects of Intravenous Patient-Controlled Analgesia with Morphine, Continuous Epidural Analgesia, and Continuous Three-in-One Block on Postoperative Pain and Knee Rehabilitation after Unilateral Total Knee Arthroplasty," Anaesthesia and Analgesia, Vol. 87, No. 1, 1998, pp. 88-92.

[4] X. Capdevila, Y. Barthelet, P. Biboulet, Y. Ryckwaert, J. Rubenovitch and F. d'Athis, "Effects of Perioperative Analgesic Techniques on the Surgical Outcome and Duration of Rehabilitation after Major Knee Surgery,” Anesthesiology, Vol. 91, No. 1, 1999, pp. 8-15. doi:10.1097/00000542-199907000-00006

[5] E. Wit and J. McClure, "Statistics for Microarrays: Design, Analysis and Inference,” 5th Edition, John Wiley \& Sons Ltd., Chichester, 2004.

[6] S. A. Strassels, C. Chen and D. B. Carr, "Postoperative Analgesia: Economics, Resourse Use and Patient Satisfaction in an Urban Teaching Hospital," Anaesthesia and Analgesia, Vol. 94, No. 1, 2002, pp. 130-137.

[7] H. K. Parvatanei, V. P. Shah, H. Howard, N. Cole, A. S. Ranawat and C. S. Ranawat, "Controlling Pain after Total Hip and Knee Arthroplasty Using a Multimodal Protocol with Local Periarticular Injections,” Journal of Arthro- 
plasty, Vol. 22, No. 6, 2007, pp. 33-38.

doi:10.1016/j.arth.2007.03.034

[8] K. Toftdahl, L. Nikolajesen, V. Haraldsted, F. Madasen, E. K. Tonnesen and K. Soballe, "Comparison of Peri- and Intraarticular Analgesia with Femoral Nerve Block after Total Knee Arthroplasty. A Randomised Clinical Trial,” Acta Orthopaedica, Vol. 78, No. 2, 2007, pp. 172-179. doi:10.1080/17453670710013645

[9] C. A. Busch, B. J. Shore, R. Bhandart, S. Gandapathy, S. J. MacDonald, R. B. Bourne, C. H. Rorabeck, R. W. McCalden, "Efficacy of Periarticular Multimodal Drug Injection in Total Knee Arthroplasty: A Randomised Trial,” The Journal of Bone \& Joint Surgery, Vol. 88, No. 5, 2006, pp. 959-963. doi:10.2106/JBJS.E.00344

[10] H. B. J. Fisher, C. J. P. Simanski, C. Sharp, F. Bonnet, F. Camu, E. A. M. Neugebauer, N. Rawel, G. P. Joshi, S. A. Schug and H. Kehlet, "A Procedure-Specific Systematic Review and Consensus Recommendations for Postoperative Analgesia Following Total Knee Arthroplasty,” Anaesthesia, Vol. 63, No. 10, 2008, pp.1105-1123. doi:10.1111/j.1365-2044.2008.05565.x

[11] P. Vendittoli, P. Makinen, P. Drolet, M. Lavign, M. Fallaha, M. Guertin and F. B. Varin, “A Multimodal Analgesia Protocol for Total Knee Arthroplasty: A Randomised, Controlled Study,” The Journal of Bone \& Joint Surgery, Vol. 88, No. 2, 2006, pp. 282-289. doi:10.2106/JBJS.E.00173

[12] Z. Dunsanka, K. Boysen, C. Christiansen, J. Christansen, S. Stephensen and B. Christensen, "A Comparison of Epidural Analgesia with Combined Continuous Femoral Sciatic Nerve Blocks after Total Knee Replacement,” Anaethesia and Analgesia, Vol. 102, No. 4, 2006, pp. 12401246. doi:10.1213/01.ane.0000198561.03742.50

[13] M. Kandasami, A. W. G. Kinninmonth and M. Sarungi, "Femoral Nerve Block for Total Knee Replacement-A Word of Caution,” Knee, Vol. 16, No. 2, pp. 98-100. doi:10.1016/j.knee.2008.10.007
[14] I. Smith, J. Hemelrijck, P. F. White, R. Shively, "Effects of Local Anesthesia on Recovery after Outpatient Arthroscopy," Anaesthesia and Analgesia, Vol. 73, No. 5, 1991, pp. 536-539. doi:10.1213/00000539-199111000-00004

[15] N. H. Badner, R. B. Bourne, C. H. Rorabeck, S. J. MacDonald and J. A. Doyle, "Intra-Articular Injection of Bupivacaine in Knee Replacement Operations. Results of Use for Analgesia and for Preemptive Blockade," The Journal of Bone \& Joint Surgery, Vol. 78, No. 5, pp. 734738.

[16] H. Ng, U. Nordstrom, K. Axelsson, A. D. Perniola, E. Gustav, L. Ryttberg, A. Gupta, "Efficacy of Intra-Articular Bupivocaine, Ropivacaine, or a Combination of Ropivacaine, Morphine, and Ketorolac on Postoperative Pain Releif after Ambulatory Arthroscoplic Knee Surgery: A Randomised Double Blind Study," Regional Anesthesia and Pain Medicine, Vol. 31, No. 1, pp. 26-33.

[17] L. J. Andersen, T. Poulsen, B. Krog and T. Nielsen, "Postoperative Analgesia in Total Hip Arthroplasty: A Randomized Double-Blinded, Placebo-Controlled Sudy on Peroperative and Postoperative Ropivacaine, Ketorolac, and Adrenaline Wound Infiltration,” Acta Orthopaedica, Vol. 78, No. 2, pp. 187-192. doi:10.1080/17453670710013663

[18] P. R. Padala, E. Rouholamin and R. L. Mehta, “The Role of Drains and Tourniquets in Primary Total Knee Replacement-A Comparative Study of TKR Performed with Drains and Tourniquet versus No Drains and Adrenaline and Saline Infiltration,” Journal of Knee Surgery, Vol. 17, No. 1, pp. 24-27.

[19] D. M. R. Gibbs, T. P. Green, C. N. Esler, “The Local Infiltration of Analgesia Following Total Knee Replacement. A Review of the Current Literature,” The Journal of Bone \& Joint Surgery Vol. 94, No. 9, 2012, pp. 11541159. doi:10.1302/0301-620X.94B9.28611 